\title{
ACTIVE COMPONENTS BASED ON DYNAMIC NEGATRONS
}

\author{
PAVLO MOLCHANOV, ARUN K. MISHRA*, \\ HELEN LINNIK and PAVLO MULYAR
}

Vinnytsia State Technical University, Ukraine

(Received 19 February 2000; In final form 25 April 2000)

\begin{abstract}
The active elements based on dynamic transistor negatrons (circuits with negative active differential resistance) are introduced. The principles of dynamic transistor negatrons simulation has been developed on the basis of non-linear charge model. Parameters, which characterize non-linear mode of dynamic transistor negatrons, are derived. Nonlinear equivalent circuit and Volterra series are used to calculate parameters of negatrons. Particular attention is devoted to the frequency mixers and frequency switches. An experimental frequency mixer has been described, that has confirms the theoretical predictions.

Keywords: Dynamic transistor negatron; Negative differential resistance; Frequency mixers; Switches
\end{abstract}

The function of amplification and generation devices is based on the use of two modes of amplification:

- amplification by means of the potential insensitive elements, (for example, electron tubes or semiconductor field effect transistors); and

- Amplification by means of the devices with negative differential resistance, which are called negatrons $[1-3]$.

Depending on function principles, the negatrons may be classified into three groups:

- Negatrons with negative resistance and $S$-mode static amperevoltage characteristics (dinisters, threenistors) (see Fig. 1a);

*Corresponding author. e-mail: arunkumarmishra@yahoo.com 
- Negatrons with negative conductivity and $N$-mode static amperevoltage characteristics (tunnel, Gun diodes) (see Fig. 1b);

- Dynamic negatrons with differential negative resistance on alternating current only (avalanche diodes and inverted common collector transistor circuits) $[3,4]$. The negative resistance in them arises by lag alternating current from the variable voltage (see Fig. 1c).

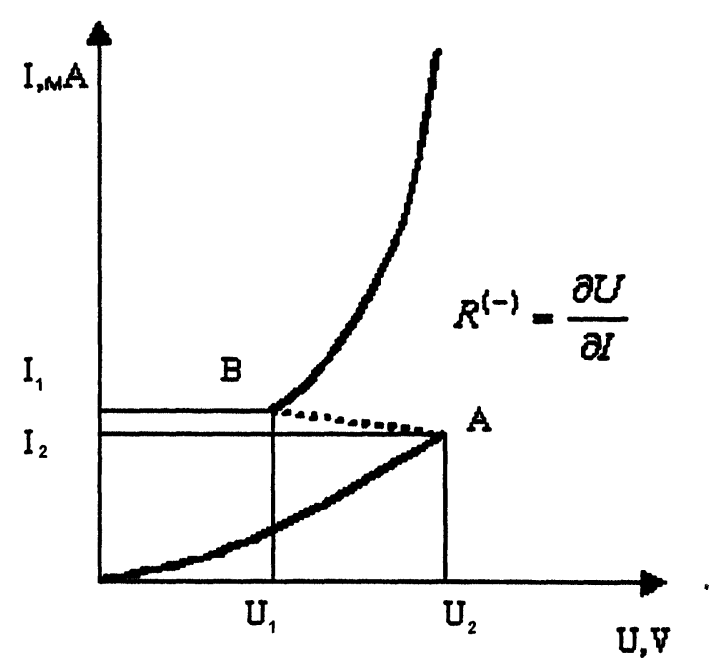

FIGURE 1a Static characteristics of $S$-type negatron.

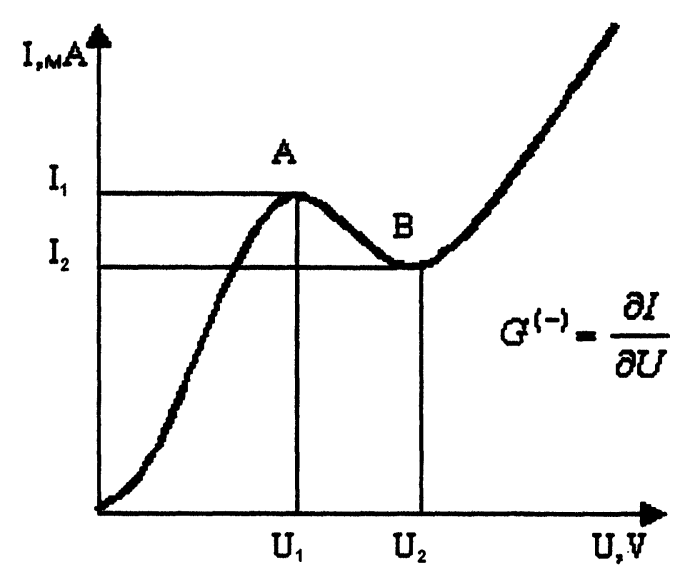

FIGURE 1b Static characteristics of $N$-type negatron. 


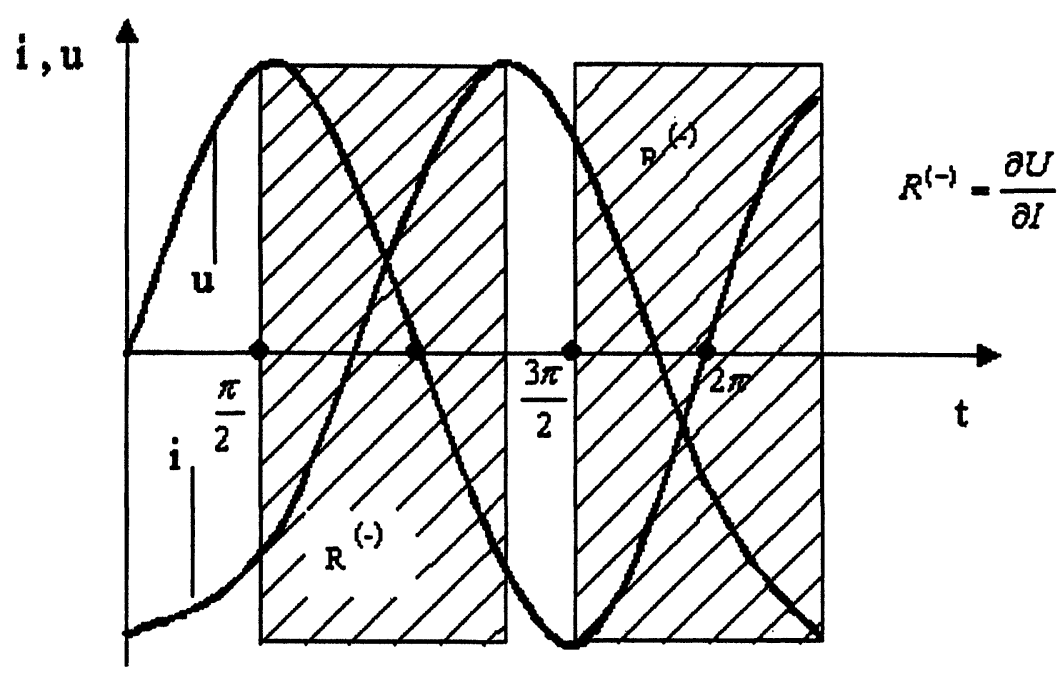

FIGURE 1c Time dependence of alternate current and voltage in dynamic negatron.

The dynamic negatrons have been used as active components for filtering, frequency multiplexing and other microwave applications [3-6]. Calculation of these circuits is based on the small-signal linear models $[1,4,5]$. But the function of such devices as oscillators and mixers is based on the non-linearity of its characteristics. The objective of this work is to present the simulation methods of active components based on nonlinear dynamic transistor negatrons. The research has been conducted in microwave range.

\section{NONLINEAR MODEL}

A nonlinear model of dynamic transistor negatrons has been developed on the basis of charge-model equations [6,7]. The suggested nonlinear model allows describing the stationary state of negatron. The non-linear equations, which define current-voltage dependencies, can be described by multiple Taylor power series, where the components, which include small-signal response, can be distinguished. Therefore the negatron can be described as a non-linear system, consisting of linear and non-linear circuits (see Fig. 2). 


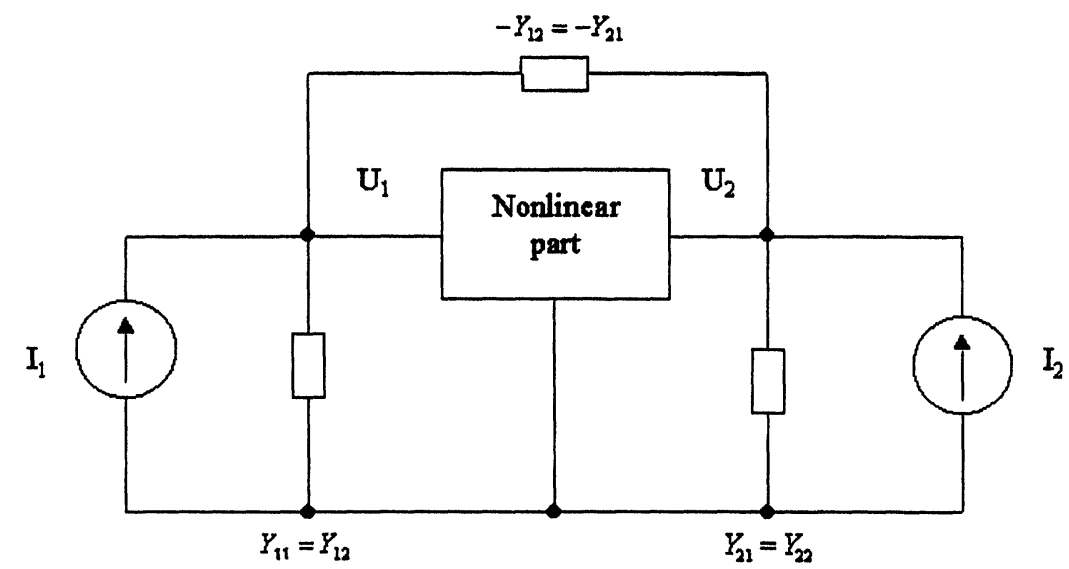

FIGURE 2 Negatron as nonlinear system, consisting of linear and nonlinear circuits.

The expressions for currents $i_{1}, i_{2}$, describe linear circuits through the convolution of pulse-periodical function (Volterra series) for the stationary mode

$$
\begin{aligned}
i_{1}= & \int_{0}^{\tau} a_{11}(\tau) \cdot u_{1}(t-\tau) d \tau+\int_{0}^{\tau} a_{12}(\tau) \cdot u_{2}(t-\tau) d \tau+ \\
& +i_{G e}(t)+\frac{d}{d t}\left[q_{e}(t)+\tau_{11} i_{G \hbar}(t)\right]-\frac{\alpha_{1} \cdot i_{G \hbar}(t)}{1+p \tau_{12}}, \\
i_{2}= & \int_{0}^{\tau} a_{22}(\tau) \cdot u_{2}(t-\tau) d \tau+\int_{0}^{\tau} a_{21}(\tau) \cdot u_{1}(t-\tau) d \tau+ \\
& +i_{G \hbar}(t)+\frac{d}{d t}\left[q_{\hbar}(t)+\tau_{22} i_{G \hbar}(t)\right]-\frac{\alpha_{N} \cdot i_{G e}(t)}{1+p \tau_{21}},
\end{aligned}
$$

where

$$
\begin{aligned}
\alpha_{N} & =\alpha_{N}\left(U_{e}, U_{\hat{e}}\right), T=\frac{2 \pi}{W} \cdot a_{11}(t)=\frac{1}{T} \sum_{l=-L}^{L} Y_{11}^{L}(j w l) e^{j l w t}, \\
a_{12}(t) & =a_{21}(t)=\frac{1}{T} \sum_{l=-L}^{L} Y_{12}^{L}(j w l) e^{j l w t} \\
a_{22}(t) & =\frac{1}{T} \sum_{l=-L}^{L} Y_{22}^{L}(j w l) e^{j l w t}
\end{aligned}
$$


$Y_{p, q}$ - Elements of conductivity matrix of the transistor's linear circuit; $T$-period of oscillation in system; $i_{G e}, i_{G}$ - currents of emitter and collector junctions respectively; $q_{e}, q$ - charges of barrier capacitance of emitter and collector junctions; $u_{1}, u_{2}$-momentary input and output voltages; $a_{N}, a_{I}$ - direct and reverse current gains; $\tau_{p, q}$ - time constant in Hamilton's model; $p$ - Laplas operator.

Expressions from (1) are divided into periodical Taylor series in domain of stationary mode. If the terms of the series are retained, then the increment components of input and output currents are written as shown in expression 2 .

$$
\begin{aligned}
d i_{1}= & \int_{0}^{\tau} a_{11}(\tau) \cdot u_{1}(t-\tau) d \tau+\int_{0}^{\tau} a_{12}(\tau) \cdot u_{2}(t-\tau) d \tau+ \\
& +q_{e} d u_{1}+\frac{d}{d t}\left[\left(C_{e}+\tau_{11} q_{e}\right) d u_{1}\right]-\alpha_{1} q_{\hbar} \frac{d u_{2}}{1+p \tau_{12}}, \\
d i_{2}= & \int_{0}^{\tau} a_{22}(\tau) \cdot u_{2}(t-\tau) d \tau+\int_{0}^{\tau} a_{21}(\tau) \cdot u_{1}(t-\tau) d \tau+ \\
& +\frac{d}{d t}\left[\left(C_{\hbar}+\tau_{22} q_{\hbar}\right) d u_{2}\right]-i_{G e} \frac{\partial \alpha_{N}}{\partial u_{2}} \cdot \frac{\partial u_{2}}{1+p \tau_{21}}- \\
& -\left(\alpha_{N} q_{e}+i_{G e} \frac{\partial \alpha_{N}}{\partial u_{1}} \cdot \frac{d u_{1}}{1+p \tau_{21}}\right)
\end{aligned}
$$

This gives us a set of differential linear equations, which connect nonlinear system in the large signal mode. If the mode is periodical, than the set of Eq. (2) passes from a nonlinear into the time-dependent linear parametric mode and may be written by the convolution in frequency range. These expressions give the linear relation between current and voltage changes and they allow calculating the transferring characteristics.

If the frequency spectrum of input excitation is limited, then the matrix equation for four-port input excitation and response are written by expressions: (3)

$$
\left[U_{c}\right]=\left[Y_{\Sigma}\right]^{-1}\left[I_{c}\right]
$$

where: $\left[Y_{\Sigma}\right]=\left[\begin{array}{ll}Y_{11} & Y_{12} \\ Y_{21} & Y_{22}\end{array}\right]$ 
- the square matrixes which responses to system of $Y$ parameters;

$$
\left[I_{c}\right]=\left[d I_{1}, d I_{2}\right]^{T}=\left[i_{-L_{1}}, \ldots, i_{01}, \ldots, i_{L_{1}}, i_{-L_{2}}, \ldots, i_{02}, \ldots, i_{L_{2}}\right]^{T}
$$

- the input current matrix vector, which describes the current change in emitter and collector circuits on the combination frequency;

$$
\left[U_{c}\right]=\left[d U_{1}, d U_{2}\right]^{T}=\left[d U_{e}, d U_{k}\right]=\left[u_{-L_{1}}, \ldots, u_{L_{1}}, u_{-L_{2}}, \ldots, u_{L_{2}}\right]^{T}
$$

- the output voltage matrix vector, which describes the voltage in emitter and collector circuits on the combination frequency;

$$
\left[Y_{p q}^{L}\right]=\operatorname{diag}\left(Y_{-L p q}, \ldots, Y_{0 p q}, \ldots, Y_{L p q}\right)
$$

- the diagonal matrix, which include the linear part of circuit's conductance in main diagonal; $\left[G_{e}\right],\left[G_{k}\right]$ - the convolution form of the differential active conduction matrix, which characterize currentvoltage junctions; $\left[C_{e}\right],\left[C_{k}\right]$ - the convolution form of the differential junctions capacitance matrix;

$$
[\Omega]=\operatorname{diag}\left(-L w+\Omega_{1}, \ldots, \Omega, \ldots, L w+\Omega\right)
$$

- the diagonal matrix of combination frequencies;

$[T]$ - the diagonal lag matrix;

$\left[G_{a e}\right],\left[G_{a \hat{e}}\right]$ - the conductance matrixes, which are the Fourier series coefficients as the functions product in time domain.

$$
p, q=1,2 ; p+q=3 \text {. }
$$

The matrix expressions (3) defines the output change for amplifying and oscillating nonlinear regime of dynamic negatrons.

\section{MEASUREMENT OF PARAMETERS}

Calculated and measured impedance components versus frequency and emitter current dependencies are shown in Figures 3-4.

The outcomes of experimental researches have completely confirmed adequacy of the proposed models. The error of measurement of negatron's parameters does not exceed $10-15 \%$ in dependence of the measurement range, and the error of simulation does not exceed $15-25 \%$. 


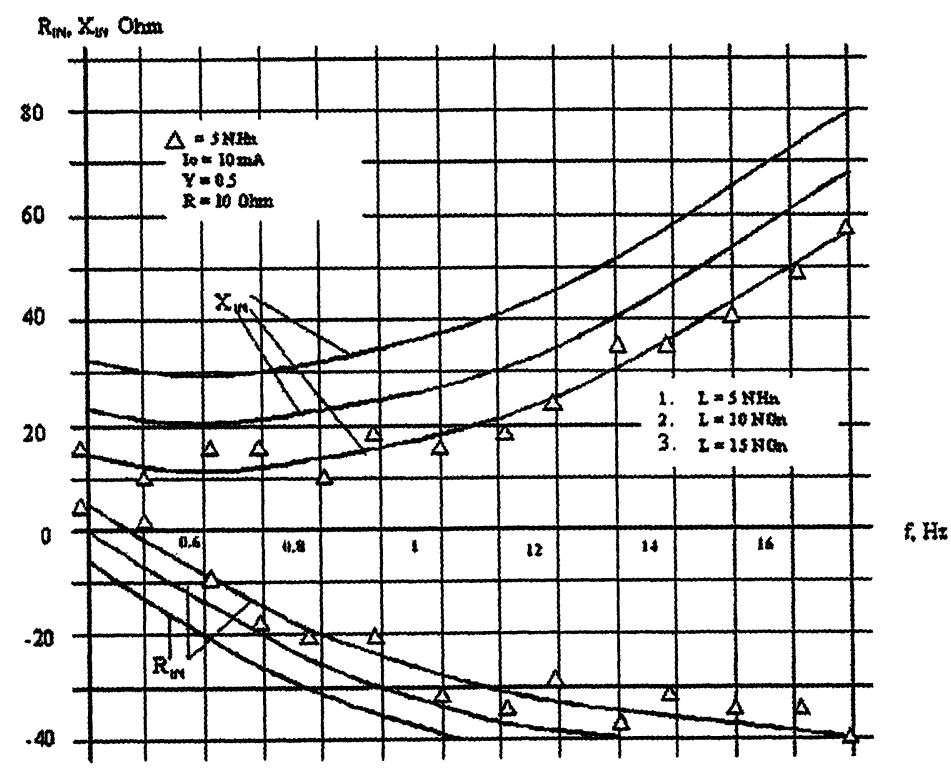

FIGURE 3 Frequency characteristics of impedance components.

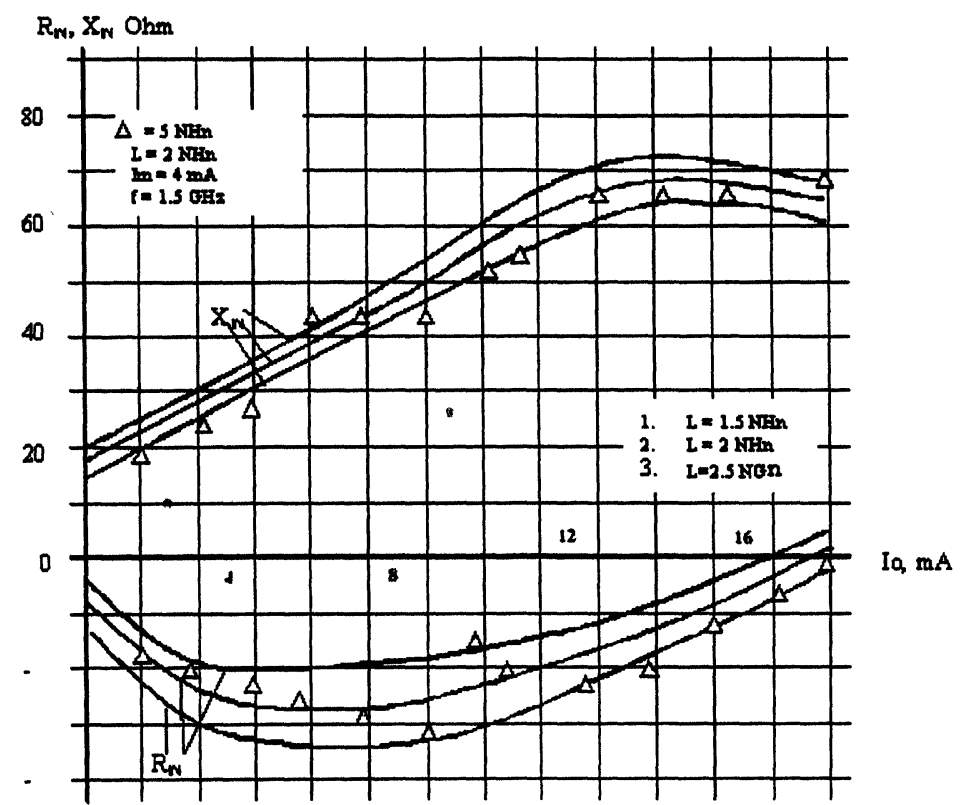

FIGURE 4 Impedance components versus emitter current characteristics. 


\section{APPLICATIONS}

The development of theoretical basis for the calculation and research of negatron's characteristics in a nonlinear regime has allowed calculating the characteristics of active components based on negatron devices. The new properties and transistor negatron's characteristics have been used for the improved performance of known devices and for development of new devices.

\section{Frequency Transformers}

The analysis of transistor negatron's work has shown, that at rather small values of amplitude of negatron's input signal, the negatron is a non-linear device which can be used as a nonlinear element of a frequency transformer. The negatrons' use for frequency transformation has two basic advantages. First, the presence of a negative resistance allows increasing the transmission factor of the circuit. Second, the use of the transistor circuits, as against application of two-poles, expands the possibilities of output

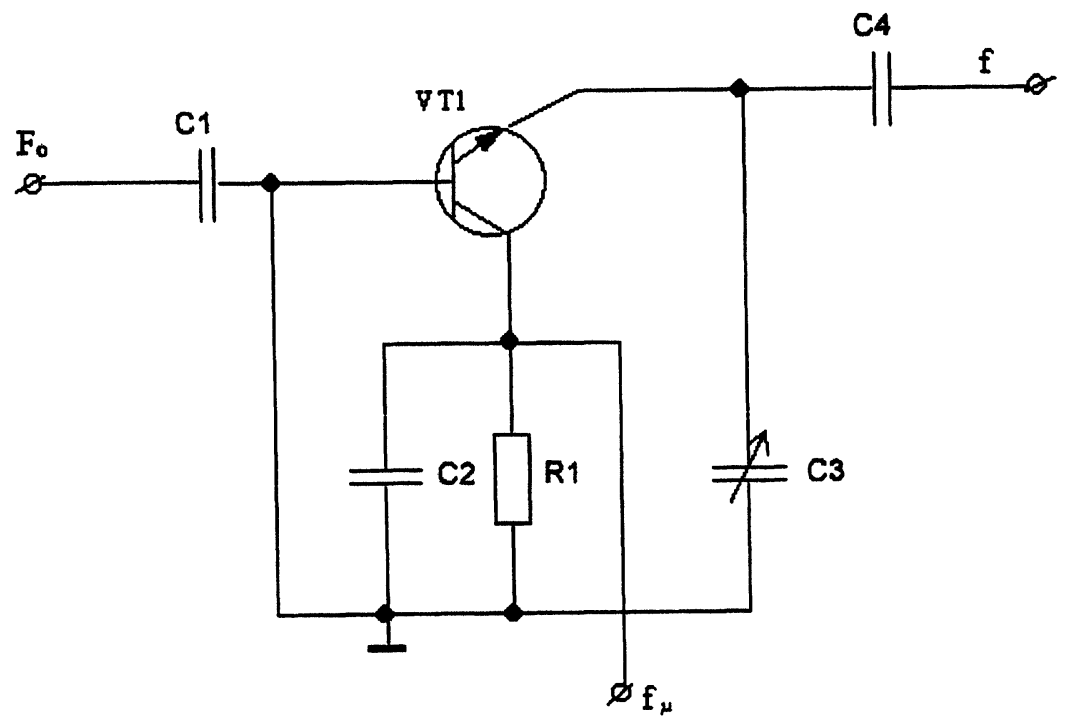

FIGURE 5 Electrical scheme of the frequency transformer on the one transistor. 
circuit, transforming signal circuit and of intermediate signal circuit de-couples.

Negatronic frequency transformer and it's characteristics shown on Figures 5-7 [6].

Reactive resistance dependence from emitter current used for frequency switcher for cellular receiver, electric scheme of which is shown on Figure 8 [8].

Active components stability been studied with the help of invariant stability coefficient. Invariant stability coefficient of transistor negatrons is calculated through scattering negatron's

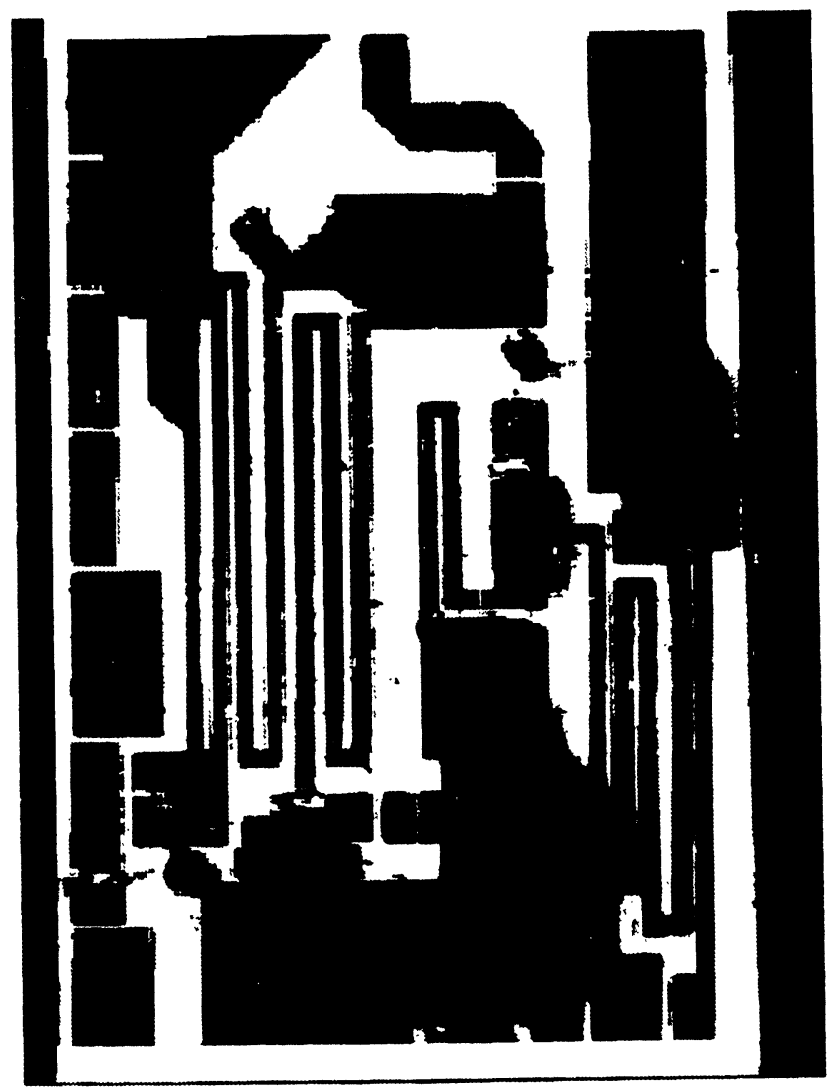

FIGURE 6 Negatronic frequency transformer. 


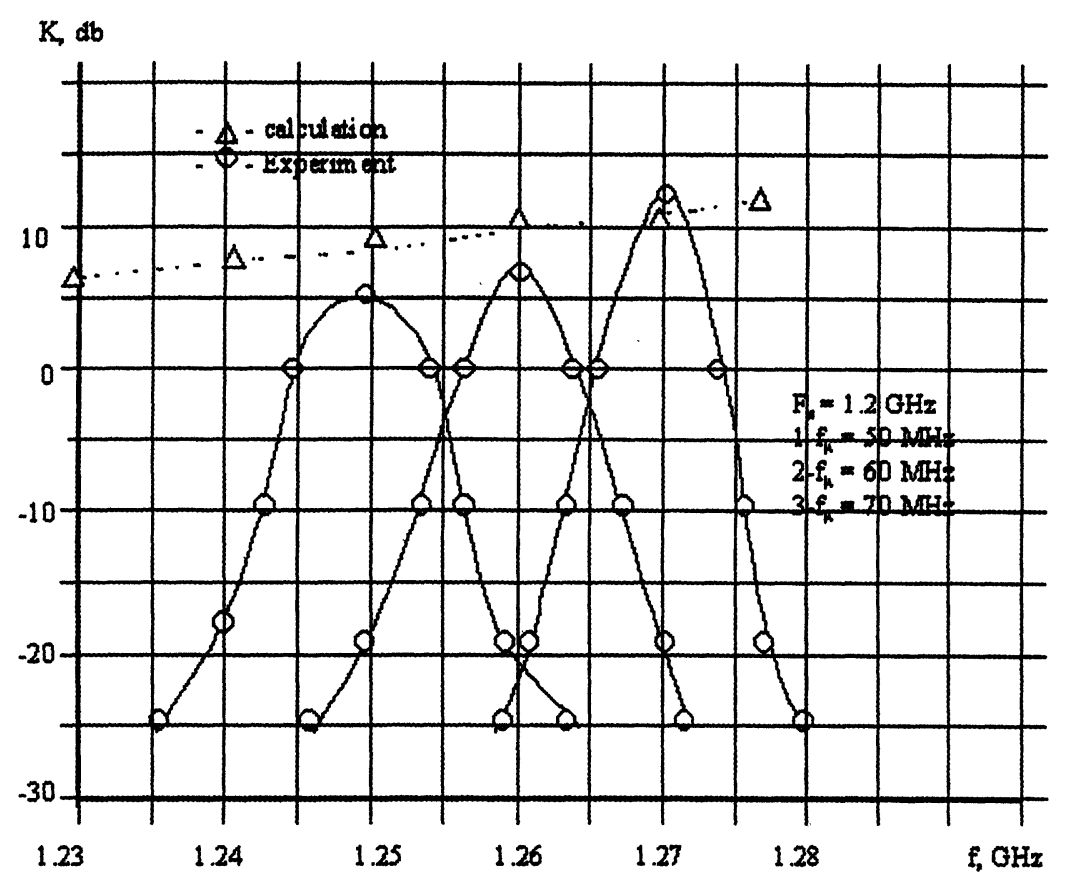

FIGURE 7 Characteristics of frequency transformer on two transistors.

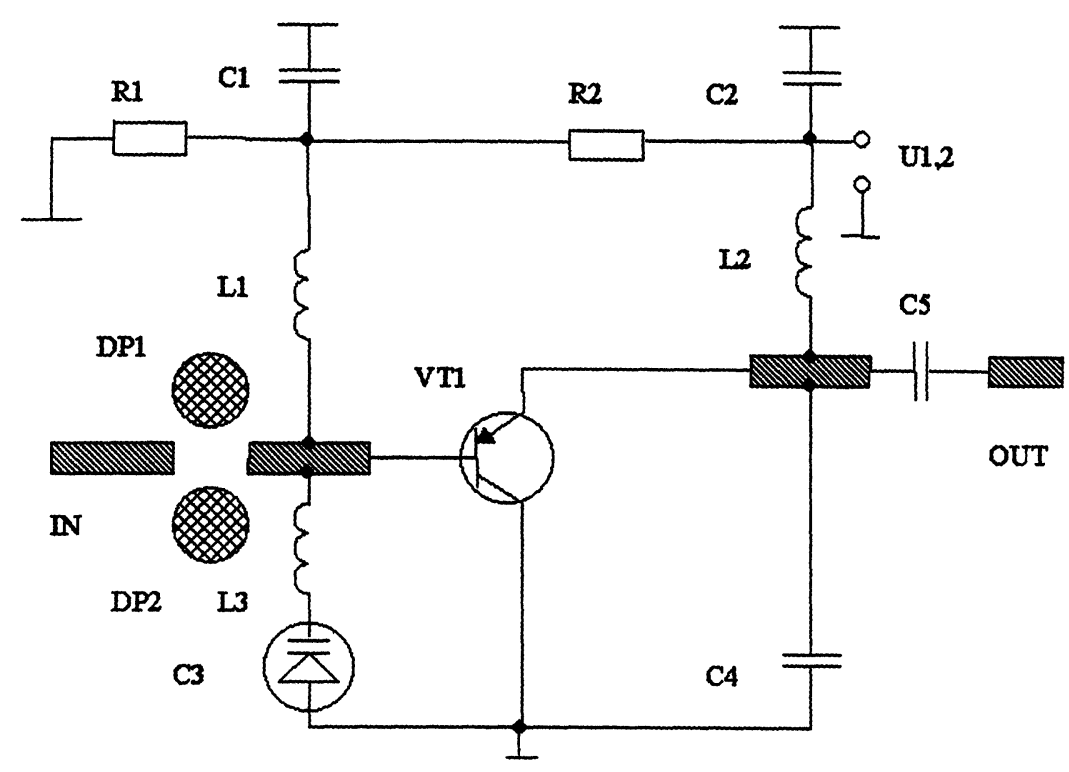

FIGURE 8 Electric scheme of frequency switcher for cellular receiver. 


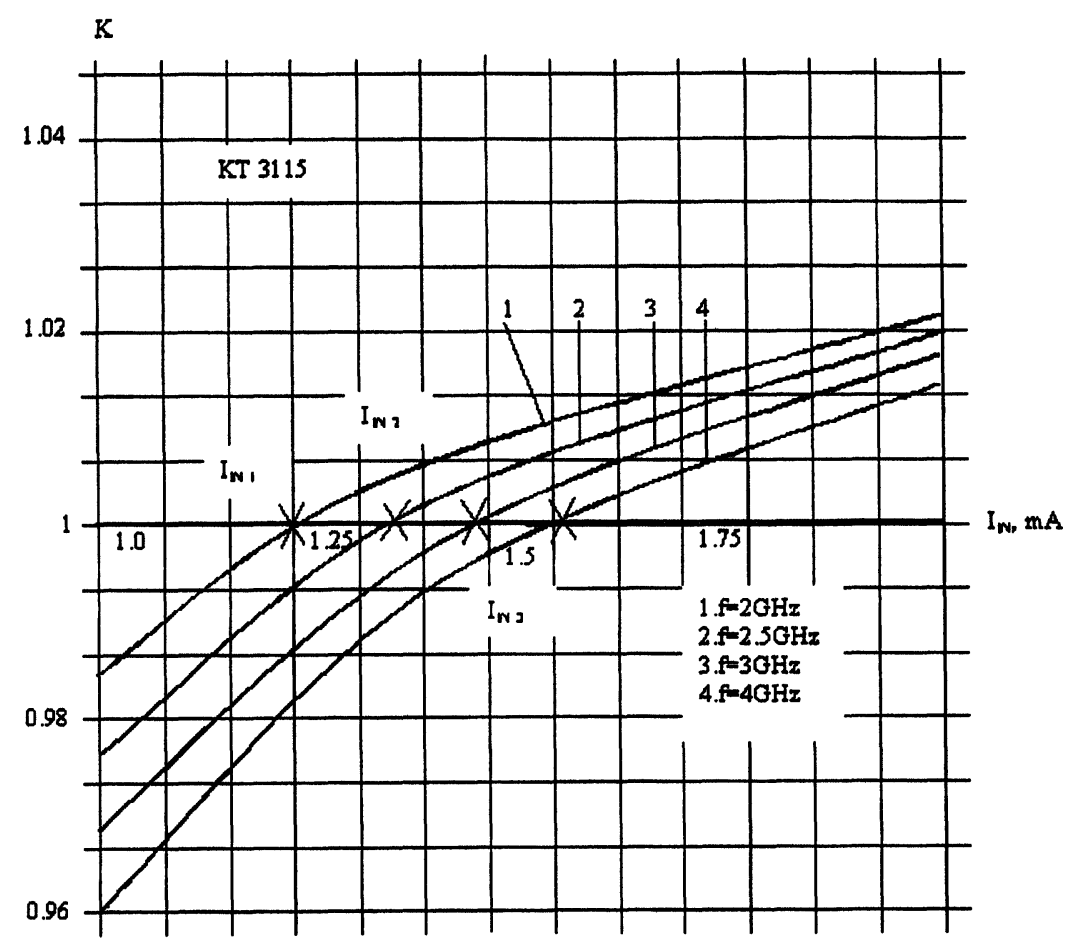

FIGURE 9 Invariant stability coefficient versus amplitude of input current characteristics.

parameters (see Fig. 9).

$$
K=1-\left|S_{11}\right|^{2}+\left|S_{22}\right|^{2}+\frac{|\Delta|^{2}}{2\left|S_{12} \cdot S_{22}\right|},
$$

where $\Delta=S_{11} S_{22}-S_{12} S_{21}$,

$S_{i j}$ - scattering negatron's parameters, which calculated through negatron's $Y$ parameters. Experiment, and 14 copyright certificates and 4 patents for the invention confirm the theoretical outcomes.

\section{CONCLUSIONS}

1. Active components based on the dynamic negatrons allow more full-use frequency properties of semiconductor devices and to do more efficiently. 
2. The non-linear models of dynamic transistor negatrons which have taken into account the influence of large signals, current, and the voltage of the input on dynamic differential negatron's parameters are described.

3. With the help of developed models, analytical relations for dynamic differential parameters of transistor negatrons in a nonlinear condition have been obtained.

4. The experimental results have enabled us to check on adequacy of the models and their analytical relations.

5. On the base of offered models the frequency transformers and frequency switching devices has been developed.

\section{References}

[1] Filinyuk, N. A., Garyinov, S. A., Seryejnov, A. N. and Stepanova, L. N., Negatronics. - Novosibirsk.: Nauka, 1995, 320 p.

[2] Molchanov, P. A., Basics of nonlinear theory of transistor negatrons. Monograph. Vinnitsa: "Universum-Vinnitsa", 1998, 207 p.

[3] Molchanov, P. A., A Nonlinear Dynamic Transistor Negatron Theory. Proceedings of NDES'99, Ronne, Denmark, July, 1999, pp. 95-99.

[4] Adams, D. K. and Ho, R. Y., Active filters for UHF and microwave frequencies. IEEE Trans. Microwave Theory Tech., MTT-17, 662-670, Sept., 1969.

[5] Chang, C. and Itoh, T., Microwave Active Bandpass Filters Using Negative Resistance Circuits. IEEE MTT-S, 38(12), Dec., 1990, 1879-1884.

[6] Molchanov, P. A., Analysis of transistor negatrons' performances in frequency transformation mode//Izv. VUZov, Radioelectronics, Kiev, Ukraine, 1998. No. 5, pp. 64-68.

[7] Molchanov, P. A., Nonlinear Model of Transistor Negatron//Electronic modeling, Kiev, Ukraine, 1998, 21(4), 21-25.

[8] A.C. 1286035 USSR, MKI-, Tunable Dielectric Resonator/Kusch, S. N., Molchanov, P. A., Dubov, E. V. and Lopato, V. G., Appl. 8.04.85; Publ. 22.09.86, Bulletin, 11 p. 

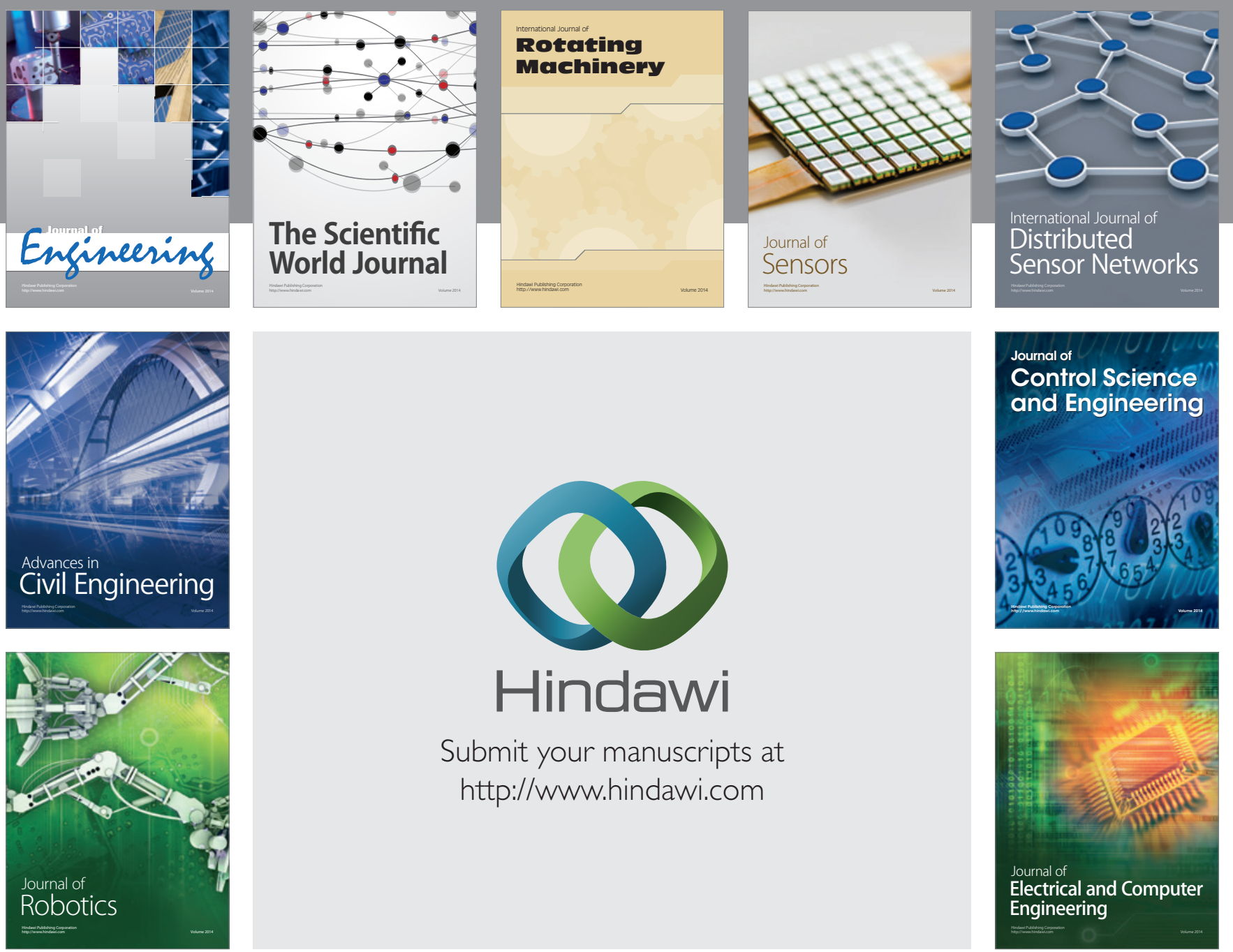

Submit your manuscripts at

http://www.hindawi.com
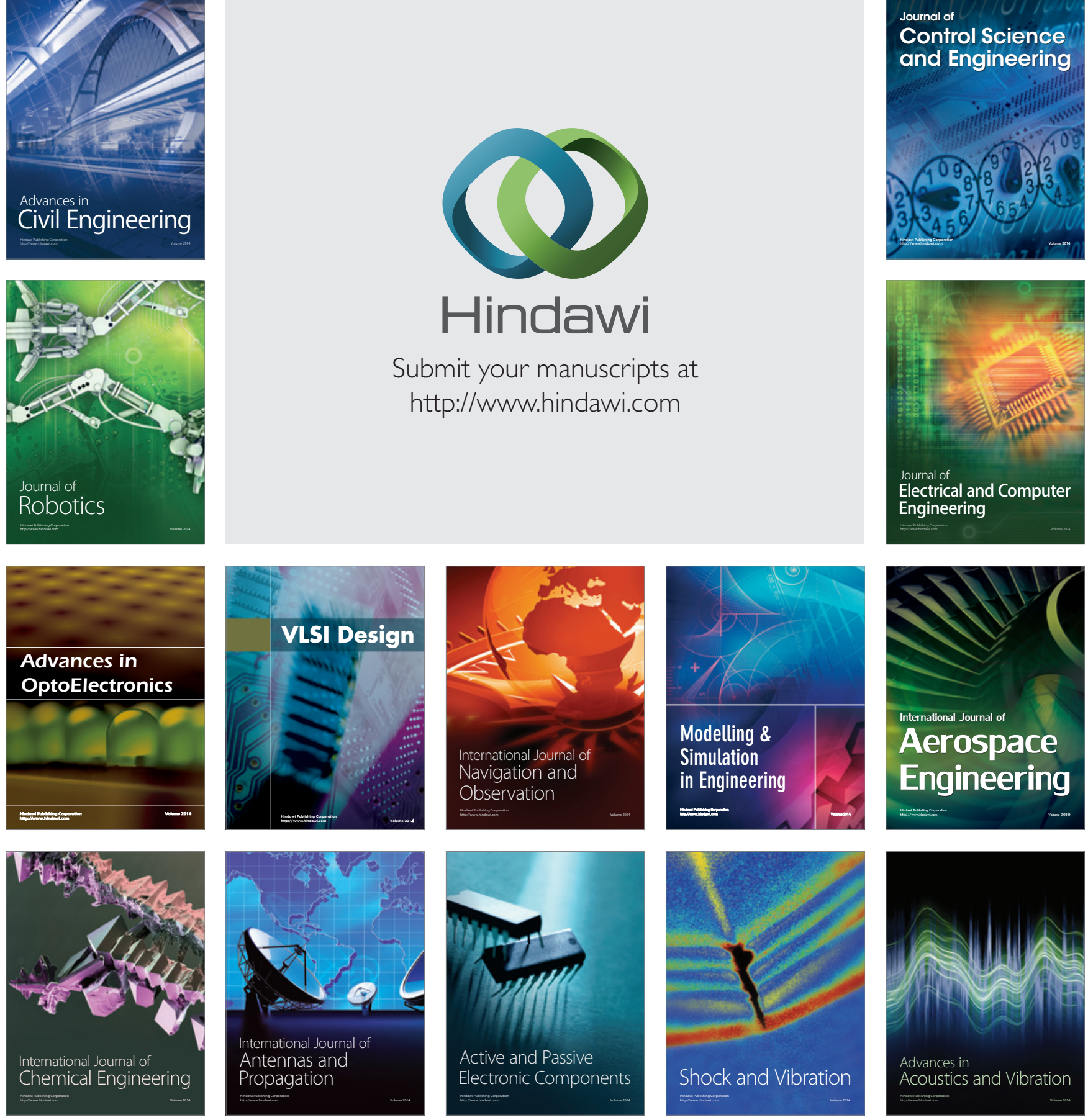